\title{
Anatomy of a Joint: Comparing Self-Reported and Actual Dose of Cannabis and Tobacco in a Joint, and How These Are Influenced by Controlled Acute Administration
}

\author{
Chandni Hindocha, ${ }^{1, *}$ Tom P. Freeman, ${ }^{1,2}$ and H. Valerie Curran ${ }^{1}$
}

\begin{abstract}
Introduction: Major gaps exist in the measurement of cannabis exposure. The accuracy of self-reported cannabis and tobacco dose per joint is poorly characterized and has never been investigated following acute cannabis/ tobacco exposure. Using an innovative "Roll a Joint" paradigm, this study aims to (1) compare estimated and actual dose of cannabis and tobacco per joint at baseline and (2) examine the acute effects of cannabis and/ or tobacco on estimated and actual dose.

Materials and Methods: We investigated this by using a randomized, double-blind, placebo-controlled crossover 2 (active cannabis, placebo cannabis) $\times 2$ (active tobacco, placebo tobacco) design in a laboratory setting Participants were 24 recreational cousers of cannabis and tobacco. At baseline, they were asked to measure out the amount of cannabis and tobacco they would put in an average joint for themselves (dose per joint). Then, on each of four drug administration sessions, participants were again asked to do this for a joint they would want to smoke "right now." Self-reported and actual amount was recorded (g).

Results: At baseline, the estimated amount of cannabis per joint $(0.28 \pm 0.23 \mathrm{~g})$ was double the actual amount $(0.14 \pm 0.12 \mathrm{~g}) \quad(p=0.003, d=0.723)$. No difference emerged between estimated $(0.43 \pm 0.25 \mathrm{~g})$ and actual $(0.35 \pm 0.15 \mathrm{~g})(p=0.125)$ amount of tobacco per joint. Compared to placebo, active cannabis reduced the actual dose of both cannabis $(p=0.035)$ and tobacco $(p<0.001)$ they put in a joint. Participants accurately estimated this reduction for tobacco $(p=0.014)$, but not for cannabis $(p=0.680)$.

Conclusions: Self-reported dose per joint is accurate for tobacco but dramatically overestimates cannabis exposure and therefore should be viewed with caution. Cannabis administration reduced the amount of cannabis and tobacco added to joints, suggesting a reduction in dose during a smoking session. The "Roll A Joint" paradigm should be implemented for better accuracy in assessing dose per joint.
\end{abstract}

Keywords: cannabis; cannabis use metrics; coadministration; dose; quantity; self-report; tobacco

\section{Introduction}

Accurate cannabis use metrics are essential for assessing the effects of cannabis. In the field of alcohol research, the concept of a "standard unit" exists as a measure of consumption. However, there is no equivalent for cannabis and self-report measures of cannabis use are at best, only weakly correlated with objective measures. ${ }^{1}$ Such metrics are used mainly as a proxy for exposure to delta-9-tetrahydrocannabinol (THC), but come with many caveats. For example, people often share cannabis and the potency and quantity of the cannabis bought, especially where sales are not tolerated,

${ }^{1}$ Clinical Psychopharmacology Unit, University College London, London, United Kingdom.

${ }^{2}$ National Addiction Centre, Institute of Psychiatry, Psychology and Neuroscience, King's College London, London, United Kingdom.

*Address correspondence to: Chandni Hindocha, Clinical Psychopharmacology Unit, University College London, 1-19 Torrington Place, London WC1E 7HB, United Kingdom, E-mail: c.hindocha@ucl.ac.uk 
are often unknown. Moreover, the quantity of use is likely to be different across types of users, where recreational users are likely to use less cannabis, and therefore potentially more tobacco, than daily users. The absence of a standardized "cannabis unit" and methodological differences between studies hinder direct comparisons regarding the effects of cannabis. ${ }^{2-5}$ Moreover, the role of frequency of use is often overemphasized at the expense of quantity (e.g., amount of cannabis used per day, per joint, or joints per gram), which is also a predictor of problematic use. ${ }^{1,6-8}$

Another major issue is that worldwide, cannabis use is strongly associated with tobacco use. ${ }^{9,10}$ Particularly in Europe, cannabis is most commonly combined with tobacco into joints as the primary consumption method. ${ }^{11}$ In Europe, "joints" typically contain a mixture of cannabis and tobacco (and are interchangeably also referred to as "spliffs"). Hereafter, we refer to joints as a mixture of cannabis and tobacco; however, we acknowledge that in the United States, joints typically do not contain tobacco. ${ }^{12}$ Adding nicotine to cannabis may modify its dose by increasing the amount of THC released by almost half ${ }^{13}$ as well as affecting the subjective experience ${ }^{14,15}$ and cognitive effects of cannabis. ${ }^{15,16}$ Combining cannabis and tobacco in joints thus exposes users to tobacco, potentially leading to a vulnerability to both nicotine and cannabis dependence. ${ }^{17,18}$ Furthermore, cannabis users titrate (adjust) their dose based on the potency of cannabis therefore modifying total THC exposure. ${ }^{19,20}$ However, thus far, whether cannabis users also titrate their dose to tobacco content has not been investigated.

Two previous studies have investigated dose per joint using a cannabis substitute. ${ }^{21,22}$ The first did not measure tobacco ${ }^{21}$; the other used the same substitute to measure both cannabis and tobacco (which have different weights) $)^{22}$ and neither was conducted with cousers who smoke joints, where this issue is particularly pertinent. ${ }^{11}$ In one Dutch study, van der Pol et al. ${ }^{1}$ estimated dose per joint using actual cannabis, however, the amount of tobacco was not estimated. Moreover, there has been no research to date investigating how acute intoxication may influence self-reported and actual dose per joint. To maximize the ecological relevance of this laboratory study, our "Roll a Joint" procedure used a typical brand of rolling tobacco and a cannabis placebo, produced from active cannabis to contain less than $0.1 \%$ THC (but with the same terpene content, so it retains the look and smells of cannabis). We first aimed to compare peoples' estimated and actual dose of cannabis and tobacco in joints. Second, we aimed to investigate how estimated and actual dose per joint is influenced by smoking cannabis and tobacco, both individually and combined in joints.

\section{Materials and Methods}

Design and participants

Participants took part in a baseline session and then four acute drug sessions. The acute effects of cannabis and tobacco, both alone and combined, were evaluated using a randomized, double-blind, placebo-controlled four-way crossover design. Previous data from this study have been published elsewhere on memory and psychotomimetic effects ${ }^{15}$ and reward processing. ${ }^{23}$ The four drug administration sessions (Table 1) were separated by at least 1 -week washout ( $\geq 3$ times elimination half-life of THC). ${ }^{24,25}$ Order of drug treatment was determined by a balanced Latin square.

Medically and psychiatrically healthy, nondependent but experienced, cannabis and tobacco cousers (i.e., "joint" smokers) were recruited from the community. Inclusion criteria were as follows: (i) 18-60 years; (ii) regular use (>once/month but $<3$ times/week) of cannabis mixed with tobacco in joints for the previous 6 months; (iii) self-reported ability to smoke one whole "standard" joint; (iv) normal or corrected to normal vision; (v) fluent English; (vi) self-reported abstinence from tobacco, cannabis, alcohol, and other drugs for at least $12 \mathrm{~h}$ before each test day; and (vii) alveolar carbon monoxide $(\mathrm{CO}) \leq 6 \mathrm{ppm}$ to confirm no recent smoking on each test day. ${ }^{12}$ Exclusion criteria were as follows: (i) $\geq 3$ on the Cannabis Severity of Dependence

Table 1. Doses for Both Cannabis and Tobacco and Their Matched Placebos Used in the Four Study Conditions

\begin{tabular}{lll}
\hline Drug & \multicolumn{1}{c}{ Condition } & \multicolumn{1}{c}{ Description } \\
\hline Cannabis & Active & $66.67 \mathrm{mg}$ Bedrobinol $(16.1 \%$ THC and $<1 \%$ CBD) \\
Tobacco & Matched placebo & $66.67 \mathrm{mg}$ placebo (Bedrocan; 0.07\% THC) \\
& Active & $311 \mathrm{mg}$ Marlboro red (15.48 mg nicotine, $16 \mathrm{mg}$ tar, $0.8 \mathrm{mg} \mathrm{nicotine} \mathrm{yield).}$ \\
& Matched placebo & $311 \mathrm{mg}$ denicotinized tobacco (Magic $0,0.04 \mathrm{mg} / \mathrm{g}$ nicotine yield)
\end{tabular}

THC, delta-9-tetrahydrocannabinol. 
$\mathrm{Scale}^{26}$; (ii) treatment-seeking for cannabis or tobacco use, or currently using nicotine replacement therapy or other cessation pharmacotherapy; (iii) smoking $\geq 10$ cigarettes a day or scoring $\geq 4$ on the Fagerström Test of Nicotine Dependence ${ }^{27}$; (iv) first cigarette smoked within the first 3 hours after waking; (v) significant respiratory, physical, or clinically diagnosed learning impairments; (vi) clinically diagnosed schizophrenia or psychosis (or a first-degree family member with either), or substance use disorder; and (vii) illicit substance use other than cannabis more than once per week.

\section{"Roll a joint" paradigm}

This paradigm was designed as an ecological assessment of participant's (a) typical dose per joint (at baseline) and (b) desired dose 1 hour after drug administration of both cannabis and tobacco. Ground placebo cannabis (which contains the precise terpene profile of the original strain, with all cannabinoids removed to $<0.2 \%$ of dry weight; available from Bedrocan NL) and rolling tobacco (Amber Leaf, JTI) were used as substitutes. Two king-sized rolling papers $(108 \times 44 \mathrm{~mm}$; Rizla Blue) were placed in front of the participant. At baseline, participants were asked to add the "amount of cannabis and tobacco they would typically put in a joint, if the cannabis and tobacco were of average strength and quality and they could smoke the whole joint by themselves." One hour after drug administration on each drug occasion, participants measured out cannabis and tobacco in the same manner. They were asked to estimate how much the amount of cannabis and tobacco they want to smoke "right now." They were then asked to estimate by sight (in g) the amount of cannabis/tobacco in each rolling paper. The weight of the cannabis and tobacco was recorded to the closest $0.01 \mathrm{~g}$. Weighing took place under experimenter-blinded conditions and participants were not given any feedback on their accuracy.

\section{Drug administration and procedure}

The effects of (a) active cannabis and active tobacco (CAN + TOB), (b) active cannabis + placebo tobacco (CAN), (c) placebo cannabis + active tobacco (TOB), and (d) placebo cannabis + placebo tobacco (PLACEBO) were investigated (doses defined in Table 1; Fig. 1).

On each drug session, participants smoked a joint (Fig. 1). The inhalation procedure was fixed and timed by an experimenter to ensure consistency between participants and sessions. Participants were asked to inhale for $4 \mathrm{sec}$, hold their breath for $8 \mathrm{sec}$, and then exhale and

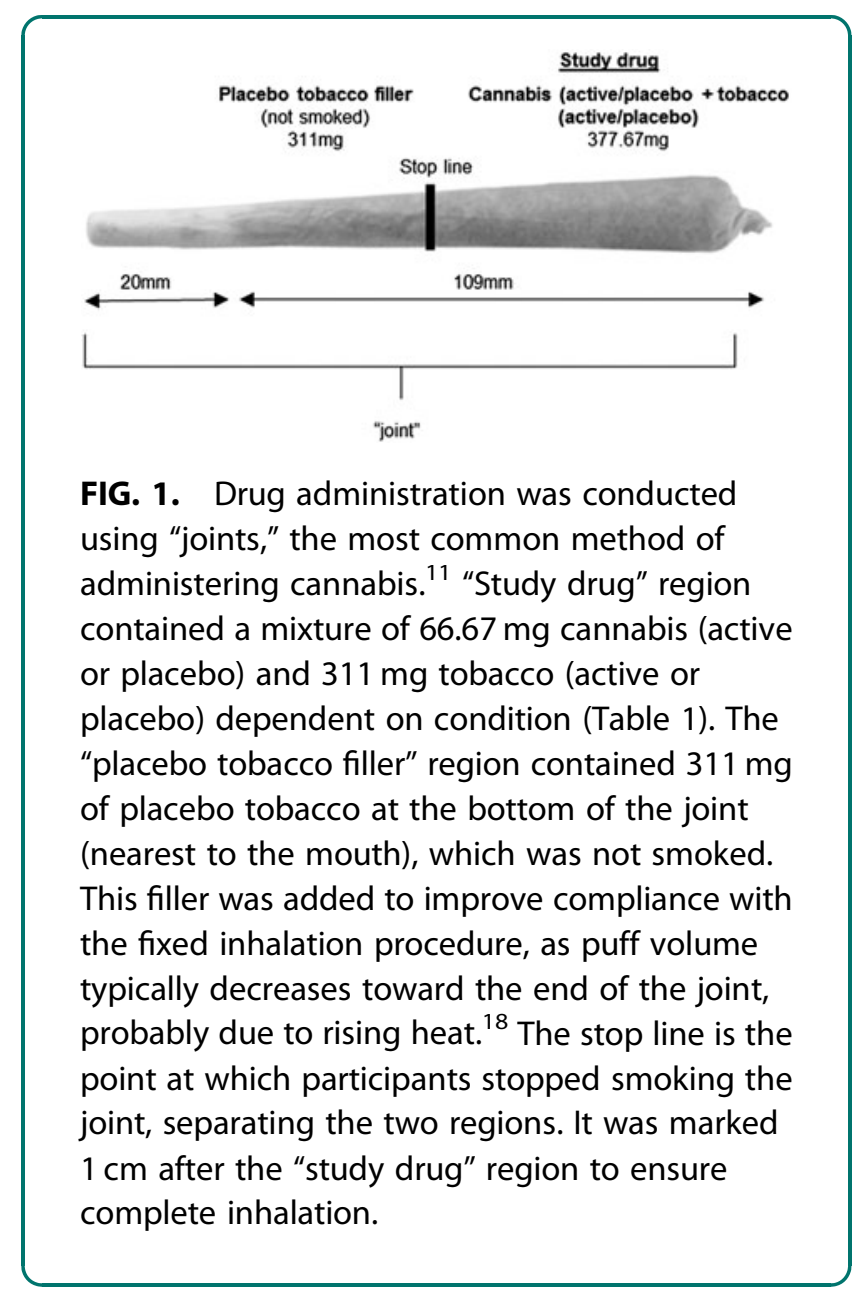

break for $30 \mathrm{sec}$. This sequence was repeated until the joint was smoked up to a designated line (Fig. 1). Participants completed the "Roll a Joint" paradigm on the baseline session and at $\sim 1 \mathrm{~h}$ after drug administration on each drug session. Visual Analogue Scale (VAS) ratings of 'stoned' (from not at all [0] to extremely [10]) were taken at $-10,+10,+30,+40$, and +70 min after drug administration. All participants provided written informed consent on each occasion. Ethical approval was given by the UCL Ethics Committee.

\section{Statistical analysis}

Bonferroni corrected paired sample $t$-tests were conducted between baseline "actual" and "estimated" dose to investigate participants' accuracy in guessing dose. Linear mixed models, with a random intercept for "participant," and two within subjects factors of cannabis (placebo; active) and tobacco (placebo; active) were implemented on both actual and estimated dose. The unstructured variance/covariance structure was selected. ${ }^{15}$ The 
dependent variable of actual and estimated cannabis and tobacco was analyzed in separate models. VAS "stoned" scores were analyzed with a cannabis (placebo, active) $\times$ tobacco (placebo, active) $\times$ time (1 [pre-drug] vs. 2-5 [postdrug] ANOVA, which used a Helmert contrast for time. ${ }^{15}$

\section{Results}

Demographics and drug history

Participants were 24 (50\% female) recreational cannabis and tobacco cousers. Use of other drugs apart from cannabis, tobacco, and alcohol was minimal (Table 2). Twenty-nine percent $(n=7)$ of participants were regular tobacco smokers before they ever mixed it with cannabis, $46 \%(n=11)$ had tried tobacco, but were not regular smokers, before mixing it with cannabis, and $25 \%(n=6)$ had never tried tobacco before it was mixed with cannabis. They self-reported adding $53.52 \% \pm 19.38 \%$ tobacco in their standard joint. They self-reported smoking skunk ${ }^{\star} 49.25 \% \pm 30.74 \%$ of the times they smoked cannabis, followed by herbal cannabis $^{\dagger}(34.7 \% \pm 28.5 \%)$ and hash ${ }^{*}(15.95 \% \pm 14.10 \%)$, respectively.

\section{Actual and estimated dose of cannabis}

\section{and tobacco per joint}

At baseline, there was a significant difference between the actual and estimated dose of cannabis participants would normally smoke $\left(t_{23}=3.36, p=0.003\right.$, $d=0.723$ ) where participants overestimated the dose twofold (Table 3). In contrast, for tobacco there was no difference between the actual dose and the estimated dose $\left(t_{23}=1.59, p=0.125\right)$.

Across each drug condition the same effect was observed, suggesting participants were accurate in estimating the amount of tobacco (all t's $\geq 0.93$, all $p$ 's $\geq 0.087$ ) but overestimated by roughly $200 \%$ the amount of cannabis used (all t's $\geq 2.62$, all $p$ 's $\leq 0.015$ ).

With regard to the actual dose of cannabis that participants rolled after intoxication, there was a main effect of cannabis $\left(F_{1,23}=5.05, p=0.035\right)$. Participants added less cannabis to their joints after smoking active cannabis $(0.12 \pm 0.03)$ compared to placebo cannabis $(0.15 \pm 0.02)$. There was no main effect of tobacco (placebo: $0.14 \pm$ 0.03 ; active $0.13 \pm 0.02$ ) or interaction with tobacco.

*Skunk refers to high-potency, indoor-grown floral material of unfertilized plants, through which energy is diverted from seed production to cannabinoid synthesis ("sinsemilla"; meaning "without seeds") ( 15\% THC). ${ }^{32}$

"Herbal cannabis refers to low-potency outdoor-grown imported floral material ("herbal," "grass," "weed") ( 9\% THC). ${ }^{32}$

"Hash refers to compressed blocks of plant matter ("resin," "hashish") ( 5\% THC).
Table 2. Demographics and Drug History of Participants

\begin{tabular}{|c|c|}
\hline & $N=24($ mean, SD) \\
\hline Female, \% & 50 \\
\hline Age (years) & $24.46 \pm 3.96$ \\
\hline SDS & $\begin{array}{c}0.67 \pm 0.92 \\
\text { (range: } 0-3 \text { ) }\end{array}$ \\
\hline FTND & $\begin{array}{c}0.33 \pm 0.64 \\
\text { (range: } 0-2 \text { ) }\end{array}$ \\
\hline \multicolumn{2}{|l|}{ Cannabis + tobacco } \\
\hline Age of first use (years) & $16.16 \pm 3.94$ \\
\hline Last used (days) & $7.92 \pm 9.64$ \\
\hline Years used (years) & $6.79 \pm 3.94$ \\
\hline Days per month & $7.75 \pm 4.43$ \\
\hline Time to smoke $3.5 \mathrm{~g}$ (days) & $36.58 \pm 34.47$ \\
\hline Lifetime exposures (days) & $627 \pm 936$ \\
\hline Exposures in the last 90 days (days) & $19.58 \pm 11.27$ \\
\hline \multicolumn{2}{|l|}{ Tobacco } \\
\hline Age of first use (years) & $15.71 \pm 1.94$ \\
\hline Last used (days) & $96.13 \pm 313.26$ \\
\hline Years used (years) & $6.76 \pm 4.58$ \\
\hline Days per month & $11.04 \pm 12.68$ \\
\hline Cigarettes per day & $2.29 \pm 2.74$ \\
\hline Lifetime exposures (days) & $2834 \pm 7202$ \\
\hline Exposures in the last 90 days (days) & $29.75 \pm 33.56$ \\
\hline \multicolumn{2}{|l|}{ Alcohol } \\
\hline Age of first use (years) & $13.12 \pm 2.40$ \\
\hline Last used (days) & $3.46 \pm 3.21$ \\
\hline Years used (years) & $9.04 \pm 4.57$ \\
\hline Days per month & $8.80 \pm 5.48$ \\
\hline Units per session & $5.88 \pm 2.68$ \\
\hline Lifetime exposures (days) & $821 \pm 557$ \\
\hline Exposures in the last 90 days (days) & $24.29 \pm 17.93$ \\
\hline
\end{tabular}

FTND, Fagerström Test of Nicotine Dependence; SDS, Severity of Dependence Scale.

With regard to the actual dose of tobacco participants rolled after intoxication, there was a main effect of cannabis $\left(F_{1,23}=22.72, p<0.001\right)$. Participants added less tobacco to their joints after active cannabis $(0.19 \pm 0.03)$ compared to placebo cannabis $(0.30 \pm 0.03)$. There was no main effect of tobacco (placebo: $0.27 \pm 0.03$; active $0.23 \pm 0.03$ ) or interaction with tobacco.

There were no main effects or interactions for the estimated dose of cannabis ( $p$ 's $>0.05$ ). However, there was a main effect of cannabis on the estimated dose of tobacco $\left(F_{1,23}=6.99, p=0.014\right)$. After active cannabis $(0.23 \pm 0.04)$, participants correctly estimated they were using a smaller dose of tobacco than after placebo cannabis $(0.36 \pm 0.05)$. There was no main effect of tobacco (placebo: $0.31 \pm 0.04$; active $0.28 \pm 0.04$ ) or interaction with tobacco.

\section{Subjective effects}

Stoned. There was a cannabis $\mathrm{X}$ time interaction $\left(\mathrm{F}_{1,23}=61.17, p<0.001, \eta_{\mathrm{p}}{ }^{2}=0.74\right)$ that revealed a significant increase between cannabis and placebo from pre- to postdrug (Fig. 2). Predrug, there was no 
Table 3. The Mean, Standard Deviation, Range, and Ratio (Cannabis:Tobacco) of the Estimated Amount and Actual Weight of Cannabis and Tobacco That Participants Rolled into a Joint During the Baseline Session and After Each Drug Condition

\begin{tabular}{|c|c|c|c|c|c|c|}
\hline & \multicolumn{2}{|c|}{ Cannabis in joint (g) } & \multicolumn{2}{|c|}{ Tobacco in joint (g) } & \multicolumn{2}{|c|}{ Ratio of cannabis to tobacco ${ }^{a}$} \\
\hline & Estimated & Actual & Estimated & Actual & Estimated & Actual \\
\hline \multicolumn{7}{|l|}{ Baseline } \\
\hline Mean & 0.28 & $0.14^{* *}$ & 0.43 & 0.35 & $0.81: 1$ & 0.53:1 \\
\hline SD & 0.23 & 0.12 & 0.25 & 0.16 & & \\
\hline Range & $0.02-0.90$ & $0.00-0.44$ & $0.10-1.00$ & $0.09-0.74$ & $0.10: 1-3.00: 1$ & $0.05: 1-1.42: 1$ \\
\hline \multicolumn{7}{|l|}{ Placebo } \\
\hline Mean & 0.32 & $0.17 * *$ & 0.38 & 0.33 & $1.12: 1$ & $0.63: 1$ \\
\hline SD & 0.29 & 0.13 & 0.26 & 0.17 & & \\
\hline Range & $0.00-1.25$ & $0.00-0.45$ & $0.00-1.00$ & $0.00-0.74$ & $0.05: 1-3.50: 1$ & $0.05: 1-2.00: 1$ \\
\hline \multicolumn{7}{|l|}{ TOB } \\
\hline Mean & 0.28 & $0.14^{*}$ & 0.34 & 0.27 & $1.22: 1$ & $0.54: 1$ \\
\hline SD & 0.28 & 0.10 & 0.26 & 0.12 & & \\
\hline Range & $0.40-1.00$ & $0.02-0.40$ & $0.00-0.90$ & $0.00-0.46$ & $0.11: 1-4.00: 1$ & $0.11: 1-1.74: 1$ \\
\hline \multicolumn{7}{|l|}{ CAN } \\
\hline Mean & 0.25 & $0.11 * * *$ & 0.24 & 0.19 & $1.23: 1$ & $0.70: 1$ \\
\hline SD & 0.20 & 0.12 & 0.20 & 0.14 & & \\
\hline Range & $0.00-0.70$ & $0.00-0.41$ & $0.00-0.60$ & $0.00-0.49$ & 0.1:1 3.33:1 & 0.05:1-2.33:1 \\
\hline \multicolumn{7}{|c|}{$\mathrm{CAN}+\mathrm{TOB}$} \\
\hline Mean & 0.32 & $0.12 *$ & 0.22 & 0.19 & $1.20: 1$ & $1.11: 1$ \\
\hline SD & 0.49 & 0.19 & 0.20 & 0.18 & & \\
\hline Range & $0.00-2.00$ & $0.00-0.59$ & $0.00-0.60$ & $0.00-0.62$ & $0.10: 1-3.00-1$ & $0.02: 1-5.50: 1$ \\
\hline
\end{tabular}

Bold text represents significant differences between estimated and actual weight. This was the case for cannabis only, and not tobacco. ${ }^{a} \mathrm{~N}$ 's range 17-24 as participants were excluded if their response was 0 for either cannabis or tobacco (as a ratio cannot be calculated). ${ }^{* * *} p \leq 0.001,{ }^{* *} p \leq 0.01,{ }^{*} p \leq 0.05$.

difference between active and placebo cannabis ( $p=0.575)$, however, active cannabis increased "stoned" ratings at all time points postdrug (all p's $<0.001)$. There was also main effects of cannabis $\left(F_{1,23}=64.97, p<0.001\right.$, $\left.\eta_{\mathrm{p}}{ }^{2}=0.74\right)$ and time $\left(F_{1,23}=151.30, p<0.001, \eta_{\mathrm{p}}{ }^{2}=\right.$ $0.87)$. There were no main effects or interactions with tobacco.

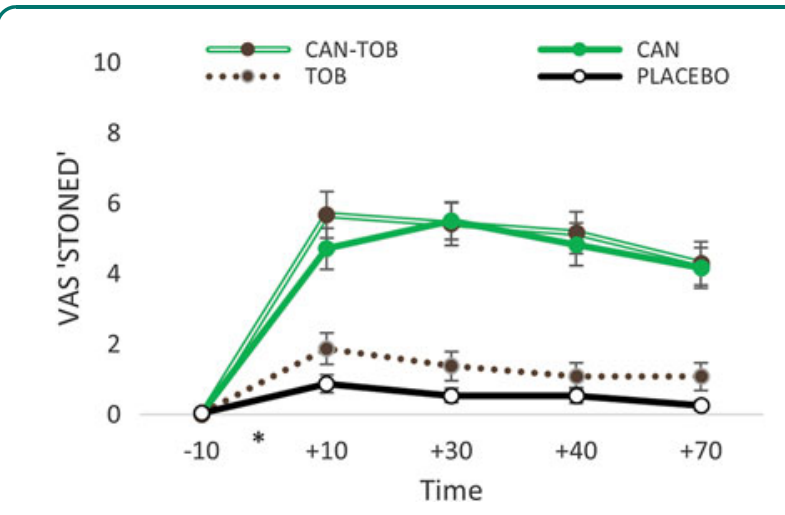

FIG. 2. VAS "stoned." Self-ratings of feeling stoned for all time points before and after each drug administration. *Represents when drug administration occurred. Error bars show \pm standard error of the mean. VAS, Visual Analogue Scale.

\section{Discussion}

This study examined estimated (self-report) and actual dose of cannabis and tobacco used in a joint. Recreational cannabis and tobacco users were assessed at baseline and after intoxication with cannabis and/or tobacco using a novel "Roll a Joint" paradigm. We used a matched placebo-cannabis and rolling tobacco to create an ecological method where their weights, smell, and appearance closely paralleled the active drugs.

Participants showed a twofold overestimation of the actual dose of cannabis they added to their joints, while accurately estimating the dose of tobacco. Importantly, this effect was replicated across all drug conditions. This suggests that overestimation of cannabis and accurate estimation of tobacco amounts are a reliable finding and impervious to acute intoxication with cannabis or tobacco. These data, alongside other studies, have found an equivalent downward titration either in the amount they rolled in joints ${ }^{20}$ or the amount they inhaled. ${ }^{1}$ For example, an Australian study that found participants overestimated the dose of cannabis to a similar degree, using a cannabis substitute, ${ }^{22}$ suggests that self-reported dose should be viewed with caution. One objective measure we would recommend is to implement this "Roll a Joint" paradigm. Given the near equivalent overestimation found in the cannabis 
amount between this study and the previous study, ${ }^{22}$ we suggest that use of a substitute, when placebo cannabis is not available, is adequate as long as a weight adjustment is made. We highly recommend that if users mix cannabis and tobacco, those tobacco estimations are made with real tobacco and recorded, unlike in previous studies. ${ }^{1,21,22}$ We encourage investigators to utilize this methodology, however, precision in dose estimation remains a problem for other routes of administration as well, and therefore, further validation of dose estimation methods is required. Given the huge variation in popular cannabis routes of administration worldwide, ${ }^{11}$ it would be necessary to validate this method for other routes (e.g., pipes, bongs, and vaporizers). There is a stark difference between the methods by which people smoke cannabis in the United Kingdom, where this study was conducted and where smoking a joint with tobacco is the most prevalent route, and the United States, where smoking cannabis through a blunt or pipe is common.

After smoking active cannabis, participants reduced both the amount of cannabis and tobacco they put into their joints compared to placebo cannabis, suggesting this paradigm is sensitive to acute satiety. Participants were only able to estimate they were using less tobacco but not less cannabis, which may imply they were aware of their satiety to tobacco, but not cannabis. This may be a consequence of the lack of information about cannabis due to nontolerated sales, which means a greater level of uncertainty regarding potency and the total weight of cannabis bought (e.g., an eighth of an ounce may not actually be what the user receives) especially for recreational users. ${ }^{20}$ Tobacco, however, is sold in standardized weights and is therefore potentially easier to estimate. Moreover, it is important to note that participants were still experiencing acute drug effects at the time of testing. Tobacco and cannabis differ in their appearance and typical dose per joint, which may have influenced accuracy; however, these factors were not manipulated and therefore are unlikely to account for the present results.

In the current turbulent climate of cannabis policy globally, finding accurate and standardized cannabis use metrics is essential to monitor levels of cannabis and tobacco consumption. ${ }^{28}$ There has certainly been some movement toward defining a standard cannabis unit $^{5}$ and certainly both frequency and quantity are important measurements. ${ }^{8}$ In Europe, the particular issue of smoking cannabis and tobacco is worrying and mostly disregarded. ${ }^{29}$ Understanding how much cannabis is in a joint will inform important drug policy discussions ${ }^{6}$ and improve research outcomes when estimating dose, especially as self-reported use is often the main outcome variable used to link cannabis consumption to health outcomes. It is essential that dose be taken into account alongside potency measures. Here we find many users were unaware of how much cannabis they put in their joints and are indeed doubling this figure. To our knowledge, this is the first study to show that both actual and estimated tobacco in joints is sensitive to acute cannabis administration indicative of crosssubstance satiety. To investigate this further, use of smoking topography would be an essential next step. ${ }^{19}$

\section{Strengths and limitations}

This study used a double-blind, placebo-controlled, four-way, crossover design with recreational users to investigate actual and estimated dose of cannabis and tobacco in a joint. A previous study found that recreational users have a poorer understanding of cannabis potency than heavy users, ${ }^{20}$ suggesting these findings cannot be extended to, and thus require replication with, heavier (dependent) users. Indeed, the precision of all dose measurement is still limited unless the potency of the cannabis is a known factor. Future research should aim to investigate dose and potency together. This research was conducted with a moderate number of recreational cannabis and tobacco users, and this may limit its generalizability (e.g., to those who do not mix their cannabis with tobacco or to those who are dependent cannabis users). Furthermore, this study was not designed to investigate sex differences and therefore was not adequately powered to address this issue. At the same time, this "Roll a Joint" paradigm has advantages over purely self-report measures of dose. We recognize this study did not have biologically verified abstinence or absorption; however, this is unlikely to influence our results as the residual cognitive effects of cannabis rarely last beyond $24 \mathrm{~h}$ and should be minimal in infrequent users. ${ }^{30,31}$

\section{Conclusions}

Self-reported dose per joint is an inaccurate cannabis use metric. Here we report that a simple, novel "Roll a Joint" paradigm can overcome these inaccuracies when collecting cannabis use metrics. Furthermore, compared to placebo cannabis, active cannabis reduces the amount of both cannabis and tobacco rolled in a joint indicative of downward titration. 


\section{Acknowledgments}

Funding was provided by a Medical Research Council $\mathrm{PhD}$ studentship to the first author. The authors thank 21st Century Ltd. for providing the denicotinized tobacco. They also thank Natacha DC Shaban for her help with drug preparation/weighing.

\section{Author Disclosure Statement}

No competing financial interests exist.

\section{References}

1. van der Pol P, Liebregts N, Graaf R, et al. Validation of self-reported cannabis dose and potency: an ecological study. Addiction. 2013;108:1801-1808.

2. Lorenzetti V, Solowij N, Yucel M. The Role of cannabinoids in neuroanatomic alterations in cannabis users. Biol Psychiatry. 2016;79:e17-e31.

3. Solowij N, Lorenzetti V, Yücel M. Effects of cannabis use on human behavior: a call for standardization of cannabis use metrics. JAMA Psychiatry. 2016;73:995-996.

4. Temple EC, Brown RF, Hine DW. The "grass ceiling": limitations in the literature hinder our understanding of cannabis use and its consequences. Addiction. 2011;106:238-244.

5. Wetherill RR, Hager N, Guthier E, et al. Gram years: a method to standardize and quantify lifetime cannabis consumption. Cannabis Cannabinoid Res. 2016;1:216-217.

6. Ridgeway G, Kilmer B. Bayesian inference for the distribution of grams of marijuana in a joint. Drug Alcohol Depend. 2016;165:175-180.

7. Walden N, Earleywine M. How high: quantity as a predictor of cannabisrelated problems. Harm Reduct J. 2008;5:1.

8. Zeisser C, Thompson K, Stockwell T, et al. A "standard joint"? The role of quantity in predicting cannabis-related problems. Addict Res Theory. 2012;20:82-92.

9. Agrawal A, Budney AJ, Lynskey MT. The co-occurring use and misuse of cannabis and tobacco: a review. Addiction. 2012;107:1221-1233.

10. Hindocha C, Shaban ND, Freeman TP, et al. Associations between cigarette smoking and cannabis dependence: a longitudinal study of young cannabis users in the United Kingdom. Drug Alcohol Depend. 2015;148:165-171.

11. Hindocha C, Freeman TP, Ferris JA, et al. No smoke without tobacco: a global overview of cannabis and tobacco routes of administration and their association with intention to quit. Front Psychiatry. 2016;7:1-9.

12. Cooper ZD, Haney M. Comparison of subjective, pharmacokinetic, and physiological effects of marijuana smoked as joints and blunts. Drug Alcohol Depend. 2009;103:107-113.

13. Van der Kooy F, Pomahacova B, Verpoorte R. Cannabis smoke condensate II: influence of tobacco on tetrahydrocannabinol levels. Inhal Toxicol. 2009;21:87-90.

14. Penetar DM, Kouri EM, Gross MM, et al. Transdermal nicotine alters some of marihuana's effects in male and female volunteers. Drug Alcohol Depend. 2005;79:211-223.

15. Hindocha C, Freeman TP, Xia JX, et al. Acute memory and psychotomimetic effects of cannabis and tobacco both "joint" and individually: a placebo-controlled trial. Psychol Med. 2017, [Epub ahead of print]; DOI: $10.1017 /$ S0033291717001222.

16. Schuster RM, Mermelstein RJ, Hedeker D. Ecological momentary assessment of working memory under conditions of simultaneous marijuana and tobacco use. Addiction. 2016;8:1466-1476.

17. Schauer GL, Berg CJ, Kegler MC, et al. Differences in tobacco product use among past month adult marijuana users and nonusers: findings from the 2003-2012 National Survey on Drug Use and Health. Nicotine Tob Res. 2016;18:281-288.

18. Patton GC, Coffey C, Carlin JB, et al. Reverse gateways? Frequent cannabis use as a predictor of tobacco initiation and nicotine dependence. Addiction. 2005;100:1518-1525.

19. van der Pol P, Liebregts $N$, Brunt $T$, et al. Cross-sectional and prospective relation of cannabis potency, dosing and smoking behaviour with cannabis dependence: an ecological study. Addiction. 2014;109:1101-1109.

20. Freeman TP, Morgan CJ, Hindocha C, et al. Just say "know": how do cannabinoid concentrations influence users' estimates of cannabis potency and the amount they roll in joints? Addiction. 2014;109:1686-1694.

21. Mariani JJ, Brooks D, Haney M, et al. Quantification and comparison of marijuana smoking practices: blunts, joints, and pipes. Drug Alcohol Depend. 2011;113:249-251.

22. Norberg MM, Mackenzie J, Copeland J. Quantifying cannabis use with the timeline followback approach: a psychometric evaluation. Drug Alcohol Depend. 2012;121:247-252.

23. Hindocha C, Lawn W, Freeman TP, et al. Individual and combined effects of cannabis and tobacco on drug reward processing in non-dependent users. Psychopharmacology (Berl). 2017, [Epub ahead of print]; DOI: 10.1007/s00213-017-4698-2.

24. D'Souza DC, Ranganathan M, Braley G, et al. Blunted psychotomimetic and amnestic effects of delta-9-tetrahydrocannabinol in frequent users of cannabis. Neuropsychopharmacology. 2008;33:2505-2516.

25. Hindocha C, Freeman TP, Schafer G, et al. Acute effects of delta-9tetrahydrocannabinol, cannabidiol and their combination on facial emotion recognition: a randomised, double-blind, placebo-controlled study in cannabis users. Eur Neuropsychopharmacol. 2015;25:325-334.

26. Gossop M, Darke S, Griffiths P, et al. The Severity of Dependence Scale (SDS): psychometric properties of the SDS in English and Australian samples of heroin, cocaine and amphetamine users. Addiction. 1995;90:607-614.

27. Heatherton TF, Kozlowski LT, Frecker RC, et al. The fagerstrom test for nicotine dependence: a revision of the Fagerstrom Tolerance Questionnaire. Br J Addict. 1991;86:1119-1127.

28. Lynskey MT, Hindocha C, Freeman TP. Legal regulated markets have the potential to reduce population levels of harm associated with cannabis use. Addiction. 2016;111:2091-2092.

29. Broyd SJ, van Hell HH, Beale $C$, et al. Acute and chronic effects of cannabinoids on human cognition-A systematic review. Biol Psychiatry. 2016;79:557-567.

30. Curran HV, Brignell C, Fletcher S, et al. Cognitive and subjective doseresponse effects of acute oral Delta 9-tetrahydrocannabinol (THC) in infrequent cannabis users. Psychopharmacology (Berl). 2002;164:61-70.

31. Curran HV, Freeman TP, Mokrysz C, et al. Keep off the grass? Cannabis, cognition and addiction. Nat Rev Neurosci. 2016;17:293-306.

32. Freeman TP, Winstock AR. Examining the profile of high-potency cannabis and its association with severity of cannabis dependence. Psychol Med. 2015;45:3181-3189.

Cite this article as: Hindocha C, Freeman TP, Curran HV (2017) Anatomy of a joint: comparing self-reported and actual dose of cannabis and tobacco in a joint, and how these are influenced by controlled acute administration, Cannabis and Cannabinoid Research 2:1, 217-223, DOI: 10.1089/can.2017.0024.

Abbreviations Used
FTND $=$ Fagerström Test of Nicotine Dependence
SDS $=$ Severity of Dependence Scale
THC $=$ delta-9-tetrahydrocannabinol
VAS $=$ Visual Analogue Scale

Publish in Cannabis and Cannabinoid Research

Cannabis and Cannabinoid Research

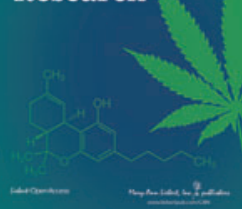

- Immediate, unrestricted online access

- Rigorous peer review

- Compliance with open access mandates

- Authors retain copyright

- Highly indexed

- Targeted email marketing 